\title{
XVIII.
}

Arbeiten ans dem Laboratorium für experimentelle Pharmakologie zu Strassburg.

\section{Ueber den Einfluss der Temperatur auf die Thätigkeit des Froschherzens.}

\author{
Von \\ Robert Flatow, \\ Arzt ans Borlin. \\ (Mit 3 Abbildungen.)
}

Das Herz des Frosehes bietet für eine grosse Reihe pharmakologiseher Untersuchungen ein vorzügliches und wichtiges Versuchsobject. Um dasselbe aber mit Nutzen zur Klarstellung der Wirkung der verschiedenen Gifte verwerthen zu können, ist es vor Allem nöthig, die physiologische Thätigkeit des Froschherzens genau zu erforschen. Besonders müssen wir alle jene äusseren Einfluisse, die infolge der Versuchsanordnung zur Wirkung gelangen, in ihrer Bedeutung für die am Herzen zu beobachtenden Erscheinungen soweit als möglich beherrsehen können. Veränderungen, die durch diese Momente in der Thätigkeit des Froschherzens hervorgerufen werden, sind naturgemäss, sobald sie nicht vorher genau bekannt sind, für pharmakologische Beobachtungen mehr oder weniger grobe Fehlerquellen.

Relativ am leichtesten übersehbar liegen ohne $Z_{w}$ eifel diese Verhältnisse bei Beobachtungen, die man an dem aus dem Organismus entfernten und künstlieh functionsfähig erhaltenen Herzen vornimmt. Die Zahl der auf die Thätigkeit des Organs einwirkenden Factoren, deren genaue Kenntniss für unsere Versuche unerlässlich ist, ist hier eine geringere; und diese selbst lassen sich eher constant erhalten. Vor Allem gilt dies von der Ernährung des am Apparat arbeitenden Herzens, die erst durch die von Williams ${ }^{1}$ ) angegebene Versuchsanordnung eine gleichmässige und ausreichende wurde. In dieser

1) Ueber die Ursache der Blutdrucksteigerung bei der Digitalinwirkung. Archiv f. exp. Path. u. Therap. Bd. XIII. 
Richtung sind zahlreiche Untersuchungen angestellt' ${ }^{1}$; andere Arbeiten berücksichtigen den Einfluss des auf dem Herzen in Systole und Diastole lastenden Druckes für die Leistungsfähigkeit desselben. ${ }^{2}$ )

Auch ein weiterer Factor, der seiner Natur nach, ebenso wie die eben genannten, bei jedem Experiment in Frage kommt, nämlich der Einfluss der Temperatur, ist schon mehrfach untersucht worden; aber hier fehlt es noch an genaueren quantitativen Feststellungen der Aenderungen, die die Temperaturunterschiede in der Herzarbeit hervorrufen. Und doch ist dies von Wichtigkeit; es wirkt ja jede Schwankung der Temperatur des Raumes, in dem man das isolirte Froschherz beobachtet, unbedingt auf die Arbeitsleistung desselben ein, da es vermöge seiner sebr geringen Masse schnell die Aussentemperatur annimmt und schon bei Veränderungen um wenige Grade - wie wir später sehen werden - nicht unbeträchtliche Modificationen seiner Thätigkeit erleidet.

Diesen Einfluss der Temperatur auf das Herz beobachteten zuerst Ernst Heinrich Weber ${ }^{3}$ ) und $B$ udge $\left.{ }^{4}\right)$. Ersterer sah das Herz des Hühnchens im $\mathrm{Ei}$ durch Wärme rascher schlagen und nahm Aehnliches auch am Froschherzen wahr. Das Letztere beobachtete auch Bindge. Später beschäftigten sich Tigges ${ }^{5}$ ) und Schelske ${ }^{6}$ ) mit der Frage. Ersterer setzte das isolirte Herz verschiedenen Temperaturen aus und bemerkte Aenderungen in der Frequenz; letzterer untersuchte hauptsächlich den bei hohen oder sebr niedrigen Temperaturen eintretenden Stillstand des Herzens und die dabei sich zeigenden Erscheinungen.

Auch Calliburcès ${ }^{7}$ ) stellte nur die Zunahme der Frequenz des Herzschlags bei Erwärmung fest. Erst $\mathrm{Cyon}^{8}$ ) beschäftigte sich ausführlich mit der vorliegenden Frage. Ihm gelang es zuerst, in Ludwig's Laboratorium eine Ernährung des isolirten Froschherzens herzustellen. Jedoch erlaubte seine Versuchsanordnung nur,

1) Vgl. Heffter, Ueber die Ernährung des arbeitenden Froschherzens. Archiv f. exp. Path. u. Therap. Bd. XXIX.

2) Vgl. Dreeser, Ueber Herzarbeit und Herzgifte. Ebenda. Bd. XXIV.

3) Muskelbewegung. Wagner's Handwörterb. der Physiologie 1846. Bd. III.

4) Sympathischer Nerv mit Rücksicht auf die Herzbewegang. Ebenda.

5) De caloris in numerum contractionum cordis efficacitate. 1853. Berlin. (Dissertation.)

6) Ueber die Veränderungen der Erregbarkeit des Herzens durch die Wärme. 1860. (Verhandlungen der naturhistorischen-medicinischen Vers. zu Heidelberg.)

7) In Claude Bernard, Système nerveux, Tome II. p. 400. 1858.

8) Ueber den Einfluss der Temperaturänderungen auf Zahl, Dauer und Stärke der Herzschläge. Berichte der sächs. Gesellsch. đ. Wissensch. 1866. 
eine ziemlich geringe Quantität Ernährungsflüssigkeit in einem künstlichen Kreislauf immer wieder in das Herz gelangen zu lassen, so dass dieselbe bald abgenutzt und untauglich wurde. Dadurch aber waren die Ernährungsverhältnisse des Organs ziemlich ungünstige.

Es ist allerdings der Einfluss der Ernährung auf die Frequenz der Pulse verhältnissmässig so gering, dass er gegenüber der bedeutenden Einwirkung, die der Temperaturwechsel austibt, ganz zuriicktritt. Anders liegt es aber für das Volum des einzelnen Herzschlags: dieses wird durch jede Veränderung der Ernährung in so hohem Grade alterirt, dass selbst die Untersebiede, die die Temperaturschwankungen erzeugen, dadurch ganz verdeckt werden können. Und somit waren Cyon's Resultate weit zuverlässiger, soweit sie die Frequenz betrafen, als für den Umfang der Contraction und folgerichtig - für die Arbeitsleistung in der Zeiteinheit.

So waren seine Forschungen, wie er selbst bemerkt, nur im Grossen und Ganzen das Gebiet erbellend, erlaubten aber nicht, die genaueren Gesetze aufzustellen.

Nur ganz nebenbei wurde die hier in Betracht kommende Frage von Blasius ${ }^{1}$ ) berührt, der lediglich Cyon's Resultate bestätigte. Den Einfluss plötzlicher Temperaturänderungen auf das Herz, den Cyon auch im Allgemeinen andeutet, behandelte Aristow ${ }^{2}$ ). Gaule ${ }^{3}$ ) prüfte die Verschiedenheiten des Verhaltens der Herzen, die von Fröschen, die im Warmen oder im Kalten gestanden hatten, entnommen waren, ohne die Temperatur der einzelnen Herzen zu variiren. Eine sehr genane Untersuchung iuber den Venensinus des Froschherzens liegt von Tigerstedt und Carlot ${ }^{4}$ ) vor, in der auch der Einfluss der Temperatur auf diesen Theil des Organs behandelt wird. Die dort angegebenen Resultate stimmen übrigens sehr gut mit den von uns für das ganze Herz gefundenen überein. Einige andere Arbeiten beschäftigen sich mit Temperatureinflïssen auf das nicht isolirte Herz und das Herz der Warmbluter und liefern so koine Ausbeute für unseren speciellen Gegenstand.

1) Am Froschherzen angestellte Versuche über die Herzarbeit unter verschiedenen innerhalb des Kreislaufs herrschenden Druckverhältnissen. Arbeiten aus d. phys. Institat d. Würzburger Hochschule. 1872. Bd.I.

2) Einfluss plötzlichen Temperaturwechsels auf das Herz und Wirkung der Temperatur überhaupt auf die Einstellung der Herzcontractionen. Archiv f. Physiologie. Leipzig 1879.

3) Die Leistungen des entblateten Froschherzens. Archiv der Physiologie. Leipzig 1878.

4) Der Venensinus des Froschherzens. Mittheilungen des phys. Labor. des medic.-chirurgischen Instituts zu Stockholm. 1888. 
Es ist somit die Frage, in welcher Weise die Temperatur die Arbeitsleistungen des Froschherzens beeinflusst, noch eine in ihren wesentlichen Theilen unbeantwortete. Und doch ist von der genaueren Durchforschung dieses Gegenstandes - freilich nicht nur am Frosch, sondern auch am Warmbluterherzen - wahrscheinlich die Lösung jener wichtigen Frage der Pathologie abhängig, ob die gesteigerte Pulsfrequenz im Fieber nur durch die Temperaturerhöhung direct hervorgerufen wird - wofür vorläufig freilich viel zu sprechen scheint.

Vor einigen Jahren hatte bereits auf Veranlassung von Herrn Prof. Schmiedeberg Herr Dr. Batten im Laboratorium des pharmakologischen Instituts zu Strassburg Versuche begonnen, um den Einfluss der Temperatur auf das isolirte Froschberz unter den Vortheilen der von Williams und Dreeser verbesserten Methodik eingehender zu erforschen. Diese Versuche gelangten indessen zu keinem Abscbluss, und so habe ich dieselben von Neuem aufgenommen.

Die folgenden Untersuchungen wurden auch in etwas anderer Weise als die früheren Batten's angeordnet. Angewandt wurde der von Williams angegebene Apparat zur Herstellung einer künstlichen Blutcirculation, und zwar in der letzten, von Dreeser verwandten Form, wie er in dessen bereits angeführter Arbeit beschrieben ist.

Zunächst erschien es als das Einfachste, nur die das Froschherz umschliessende Glaskapsel in ein Gefäss mit verschieden zu temperirendem Wasser zu setzen. Man musste sich aber sagen, dass so das in das Herz einfliessende Blut eine etwas andere Temperatur haben könne, als das Organ selbst. Das Blut in den Kugeln, aus denen es in den künstlichen Kreislauf eintritt, zu erwärmen, erschien anch nicht zweckentsprechend; denn ein Vorversuch ergab, dass sich das Blut auf dem relativ kurzen Wege vom Reservoir zum Herzen infolge der ausserordentlich geringen Durchflussgeschwindigkeit um mehrere Grade abkühlt.

Deshalb entschlossen wir uns, den ganzen Apparat derart in ein geräumiges Wasserbad mit gläserner Seitenwandung zu setzen, dass nur noch die Oeffnnngen der Zuflussgefüsse das Wasserniveau überragten. Zwei hochstehende Gefässe, das eine mit kaltem, resp. Eiswasser, das andere mit warmem Wasser gefüllt, wurden durch Heber mit dem Wasserbad in Verbindung gebracht, so dass bequem und rasch jeder gewünschte Wärmegrad in demselben hergestellt werden konnte.

Nach Dreeser's Vorgang wurde das Herz in eine Kapsel mit gut schliessendem Korke gebracht, der jedoch für den vorliegenden 
Zweck mit drei Durehbohrungen versehen war. Durch eine der Oeffnungen führte das den Kreislauf mit der Kronecker'schen Canüle, resp. dem Herzen verbindende Röhrchen (vgl. Fig. 1, a), durch die zweite ein kleines Thermometer (b), dessen Kugel sich in gleicher Höhe mit dem Herzen befand. In die dritte endlich wurde eine T-förmig gebogene Glasröhre eingeführt. Der eine Schenkel (c) derselben bildete ein enges Rohr, das sich vor einer horizontalen. Scala befand, zur Ablesung der Schwankungen des Volums der in der Kapsel eingeschlossenen Flüssigkeit bestimmt, das Volumeter. Um den Apparat für das Einsetzen und Herausnehmen aus dem Wasserbad handlich zu machen, wurde das Volumeterrohr möglichst kurz

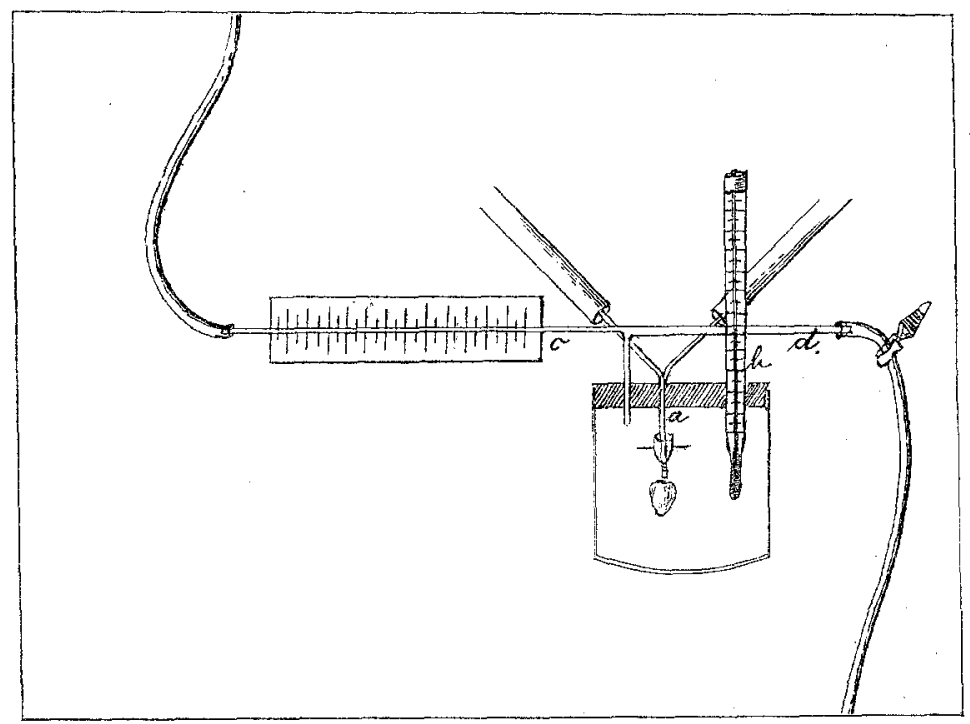

Fig. 1.

$(8 \mathrm{~cm})$ gewählt. Bei längerer Dauer der Versuche und bei Anwendung höheren Druckes trat jedoch selpst bei gut functionirenden, nicht blutenden Herzen (nur solche lieferten natürlich brauchbare Resultate) allmählich etwas Serum aus dem Herzinnern und vermehrte das Volum der in der Kapsel enthaltenen Flüssigkeit, so dass sich der Anfang der Flïssigkeitssäule im Volumeter vom Nullpunkt um einige Millimeter verschob. Vor Allem aber musste man auch darauf Rücksicht nehmen, dass beim Erwärmen und Abkühlen der Kapsel das Volum der in ihr vorhandenen Flüssigkeit sich nicht unbedentend veränderte, da ziemlich weit auseinanderliegende Temperaturen Anwendung fanden. So wurde es während der. Versuche öfters noth- 
wendig, die Flüssigkeitssäule im Volumeter wieder auf den Nullpunkt einzustellen. Daher wurde der zweite Schenkel der aus der Kapsel herausführenden T-röhre $(d)$ mit einem dünnen Gummischlanch verbunden, der durch den Boden des umgebenden Wasserbades geführt war. Anch das Scalenrohr war natürlich durch einen uber den Flüssigkeitsspiegel geführten Schlauch mit der äusseren Luft in Verbindung. Nachdem das Herz eingebunden war, wurde die Kapsel und die damit in Verbindung stehenden Röhren ganz mit NaCl-Lösung gefüllt, und der nach unten fübrende Schlauch dicht an der T-röhre zugeklemmt. Das Zurtickbleiben von Luftblasen kann man dabei leicht vermeiden, wenn man diese durch die Durchbohrung für das Thermometer entweichen lässt und dies zu allerletzt einführt. Durch Oeffnen der Klemme war es, infolge der Heberwirkung dieses Ansatzstückes, bequem möglich, Flüssigkeit abzulassen und die Säule auf den Anfangspunkt der Scala einzustellen.

Zur Ernährung wurde die von Dreeser angegebene und von Heffter als sehr zweckmässig gefundene Flüssigkeit benutzt $(1 / 3$ defibrinirtes Rinderblut mit 2/3 physiologischer Kochsalzlösung), ein ganz vorzügliches Nährmaterial, in dem die Herzen viele Stunden lang ihre ursprüngliche Leistungsfähigkeit bewahrten. Auch in die das Herz umgebende Kapsel wurde diese Flüssigkeit gefüllt, sobald nichts daran lag, das Herz selbst zu beobachten: vermöge ihrer rothen Farbe erleichtert sie sehr die Ablesung des Volums unter Wasser.

Die Versuche wurden alle mit Esculentaherzen angestellt.

Die Pulszahl wurde mittelst eines Chronometers direct beobachtet.

Das Volumen der bei jedem Pulsschlage ausgeworfenen Blutmenge wurde theils direct durch Wägung der darch je 20 Contractionen geförderten Blutmenge bestimmt, theils durch Ablesung der Verschiebung der Blutsäule an der Scala ermittelt. Beide Arten der Bestimmung ergaben, wie ich mich wiederholt überzengte, durchaus gleichwerthige Resultate. Denn ein Theilstrich unserer Scala entsprach stets fast genau einer vom Herzen ausgeworfenen Blutmenge von $0,005 \mathrm{~g}$.

Zwei Vorsichtsmaassregeln stellten sich bei den Versuchen bald als nothwendig heraus. Zunächst mussten wir für Versuche, die das Volumen der Pulse ergeben sollten, ganz von der Bestimmung der absoluten Kraft absehen. Es zeigte sich nämlich, wie schon Dur$\mathfrak{d} \mathfrak{u} \mathrm{fi}^{1}$ ) andeutet, dass starke Ueberlastungen, wie man sie bei Untersuchungen der gedachten Art anwenden muss, von bedeutendem und

1) Beiträge zur pharmakologischen Physiologie des Froschherzens. Archiv f. exp. Path. u. Therapie. Bd. XXV. 
Ueber den Einfluss d. Temperatur auf die Thätigkeit des Froschberzens. 369

langdauerndem Einfluss auf die Herzcontractionen waren. Sie wirkten nämlich so ermüdend auf die Musculatur, dass nur nach langer Zeit und oft überhaupt nur unvollkommen der ursprüngliche Zustand des Herzens sich wiederherstellte. So wurden diese Bestimmungen einer besonderen Versuchsreihe zugewiesen.

Zweitens wirken, wie Cyon und ausführlicher Aristow (l. c.) gezeigt, plötzliche Aenderungen der Temperatur auf Frequenz und Umfang der Herzcontractionen eigenthümlich ein, was man wohl als eine vorübergehende Reizerscheinung auffassen muss. Es ergaben sich auch bei unseren Versuchen beim Uebergang von einer Temperatur zur anderen zunächst besondere Aenderungen in der Frequenz und im Volum, sogar Unregelmässigkeiten, die erst nach einiger Zeit verschwanden. So wurde dann stets folgendermaassen verfahren.

Die Temperatur des Wasserbades wurde auf den gewünschten Wärmegrad gebracht und genau erhalten. Das Thermometer neben dem Herzen, ein zweites in den Zuflusskugeln und ein drittes, das die Temperatur des Wassers anzeigte, wurden beobachtet und darauf geachtet, dass alle drei den gleichen Grad zeigten. 15 Minuten, nachdem Herz und Blut sich bis zu der betreffenden Temperatur erwärmt hatten, wurde die Ablesung vorgenommen.

Unter Berücksichtigung aller genannten Verhältnisse ergaben die in angegebener Weise ausgeführten Versuche die im Folgenden wiedergegebenen Resultate.

Zunächst wurde der Einfluss der Temperatur auf die Frequenz und gleichzeitig auf das Volum in einer grösseren Zahl von Versuchen festgestellt, und es zeigte sich dabei hinsichtlich der Pulsfrequenz Folgendes. Das Minimum der Frequenz liegt bei niederen Temperaturen, das Maximum bei hohen. Die Temperatur von $0^{0}$ habe ich wegen der Schwierigkeit, sie bei der Sommerwärme, bei der ich arbeitete, constant zu erhalten, nicht angewandt. Cyon sah bei Temperaturen zwischen $0^{0}$ and $4^{0}$ Stillstand des Herzens eintreten. Batten hatte bei $0^{0} 4$ Schläge beobachtet. Ich fand bei $3^{0}$ durchschnittlich 8 Schläge. Aus dem Verlanf der ïbrigen Curven lässt sich auch berechnen, dass die schliesslich ganz langsame Schlagfolge bei $0^{0}$ in völligen Stillstand übergeht.

Von dem Punkte der langsamsten. Schlagfolge an nimmt nun die Frequenz der Herzschläge, im Ganzen regelmässig, mit der Temperatur von Grad zu Grad zu. Bis zu einer Temperatur von ungefähr $10^{\circ}$ beträgt die Zunahme etwa pro Grad 2-3, von $10^{\circ}$ an schwankt sie zwischen 3 und 5 und beträgt also im Mittel ungefähr 4 pro Grad. 
Des Vergleiches wegen will ich nur anfuhren, dass Lie ber m e i ster bei den Temperatursteigerungen des fiebernden Menschen eine $\mathrm{Za}$ nahme der Palsfrequenz um 8 Schläge pro Grad fand.

Das Verhalten der Frequenz der Schläge des Froschherzens bei verschiedenen Temperaturen ist aus der beigefügten Curve ersichtlich (vgl. Fig. 2).

Die Figur giebt das Resultat eines Versuches wieder. Als Abscissen sind die Temperaturen aufgetragen, als Ordinaten die verschiedenen an einem Herzen beobachteten Werthe. Die zahlreichen Versuche mit anderen Herzen ergaben fast gleiche Curven, so dass die Wiedergabe der einen wohl genügt. Natürlich wurden immer andere Temperaturen zur Ausführung der einzelnen Bestimmungen gewählt, da jedes Herz nur eine gewisse Anzahl ohne Schädigung ertrug.

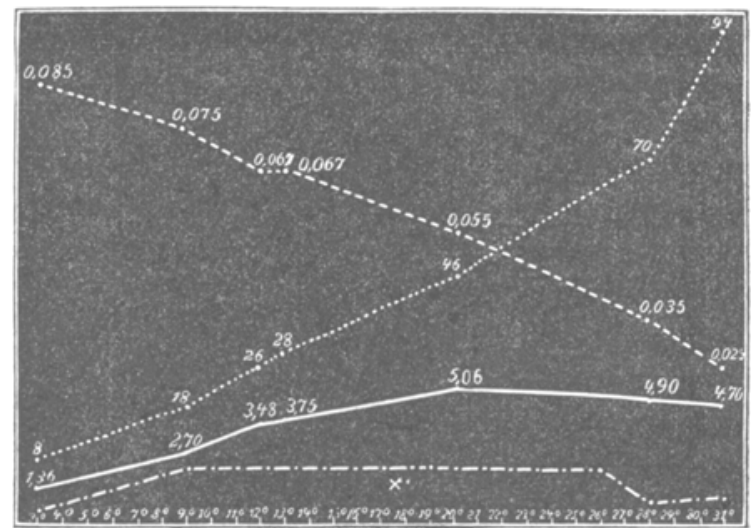

Fig. 2.

Die Zahlen unten geben die Temperatur des Herzens an.

Die Punkte bezeichnen die Grade, bei denen die Messungen stattgefunden haben.

Curve der Frequenz der Herzschläge.

Die Zahlen geben die Anzahl der Schläge in der Ninute an.

Curve der Volumina der Pulse.

Die Zahlen geben das von einem Puls ansgeworfene Blutquantum in Cubikcentimetern an.

Curve der Arbeitsleistungen des Herzens in der Zeiteinheit.

Die Zahlen geben die Arbeitsleistung in der Minute in GrammCentimetern an.

_...... Curve der absoluten Kraft. X Letzte Messung derselben nach dem Erwärmen und Abkühlen.

Die punktirte Linie stellt die uns hier interessirende Pulsfrequenz dar. Diese Curve zeigt also einen fast geradlinigen Verlauf.

Bemerkenswerth ist übrigens, dass die absoluten Zahlen der Herzfrequenz bei derselben Temperatur auch für verschiedene Individuen nahezu die gleichen sind. Wie wir später sehen werden, ist 
Ueber den Einfluss d. Temperatur auf die Thätigkeit des Froschherzens. 371

dieses Verhältniss bei der Grösse der Pulsvolumina und der Arbeitsleistungen ein bedeutend anderes.

Als Durchschnittswerth kann man wohl für eine Temperatur von $20^{\circ}$ etwa 60 Contractionen in der Minute rechnen, was nach den obigen Regeln für $10^{\circ}$ etwa 20 Pulse, für $30^{0} 100$ ergiebt, welchen Zahlen die bei der Mehrzahl der Versuche beobachteten Werthe recht gut entsprechen. Als absolut höchste Frequenzziffer habe ich 120 bei $32^{\circ}$ erbalten, wobei freilich zu bemerken, dass in diesem Fall die Regelmässigkeit des Rhythmus nicht völlig gewahrt war. Vergleicht man die einzelnen Versuche hinsichtlich der proportionalen Schlagzahlen unter einander, so ist die Uebereinstimmung eine vollständige.

Was nun das obere Maximum der Temperaturgrade betrifft, bei denen das Herz noch arbeitet, so liegt dasselbe in der Mehrzahl der Fälle bei $32^{\circ}$, gelegentlich auch bei $31^{\circ}$ und noch etwas tiefer, znweilen auch ein wenig höher. Bei höheren Temperaturen stellt dann das Herz seine Thätigkeit ein, wenigstens wenn es längere Zeit darin verweilt. Ganz kurze Zeit $\left(3^{\prime}\right)$ vermag es aber auch nach Aristow (l. c.) Temperaturen bis $40^{\circ} \mathrm{zu}$ ertragen. Auch wenn es eben aufgehört bat, zu schlagen, gelingt es nach Aristow durch schnelles Abkühlen, es wieder functionstüchtig zu machen. Andere Beobachter, wie Cyon, geben freilich an, dass das Maximum der Frequenz bei einer niedrigeren Temperatur liege, und dass dann, bei weiterer Erwärmung, die Pulsfrequenz wieder und zwar sehr erheblich sinkt. Nach meinen Versuchen ist der Sachverhalt der, dass die Frequenz unablässig bis zum Maximum steigt, und dann auf dieses der Stillstand unmittelbar folgt. Aber freilich schon mehrere Grade, ehe das Maximum erreicht ist, oft schon bei $28^{\circ}$, stellen sich Unregelmässigkeiten derart ein, dass ab und zu einzelne Schläge ausfallen. Zählt man nun blos die Zahl der im Volumeterrohr sichtbaren Contractionen, so erhält man eine niedrigere oder höchstens dieselbe Pulszahl, wie bei der vorhergehenden Beobachtung bei einem niedrigen Temperaturgrad. Rechnet man aber stets die ansfallenden Contractionen, entsprechend dem allgemeinen Rhythmus, mit, so erhält man mit der höheren Temperatur stets entsprechend ansteigende Zahlen für die Pulsfrequenz. Je höher die Temperatur gesteigert wird, um so mebr Schläge fallen allerdings aus, aber der allgemeine Rhythmus wird doch ein entsprechend schnellerer, bis endlich nach einer kurzen Periode sich sebr schnell folgender Pausen der völlige Stillstand wirklich eintritt. Häufig sieht man sowohl am isolirten wie an dem in situ befindlichen Herzen bei diesen Grenztemperaturen die Vorhöfe noch unbeirrt den entsprechend schnellen und gleichmässigen 
Rhythmus einhalten, während am Ventrikel schon einzelne Contractionen ausfallen. Es macht in diesem Falle den Eindruck, als ob der Ventrikel wohl den Impuls zur Contraction empfänge, seine Musculatur demselben aber nicht nachzugeben vermöge. Wir geben hier

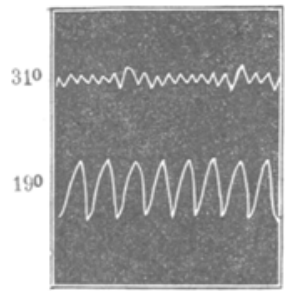

Fig. 3. zwei durch ein $\mathrm{Hg}$-Manometer gewonnene Curven der Herzcontractionen wieder, von denen die eine bei normaler Temperatur $\left(19^{\circ}\right)$, die andere bei einer Temperatur von $31^{\circ}$ von demselben Herzen aufgeschrieben wurde. Es entspricht die letztere Curve dem Stadium, in welchem die gesteigerte Frequenz deutlich hervortritt, und das Ausfallen einzelner Scbläge gerade beginnt. In diesem Falle beträgt die Frequenz bei $19^{\circ}$ in der Minute 48 Schläge, bei $31^{\circ}$ dagegen 112 , sofern man in die Pausen die Schläge interpolirt; unterlässt man dies dagegen, so erhält man dann allerdings nur etwa 100 Schläge pro Minute.

Es liegt die Frage nahe, ob diese Beobachtung nicht vielleicht mit der am fiebernden Menschen gemachten Erfahrung in Zusammenhang gebracht werden kann, dass bei höheren Temperaturen eine dieser vergleichbare Arbythmie, ein Ausfallen einzelner Pulse, gelegentlich auftritt.

Wenden wir unsere Aufmerksamkeit nun weiter der von dem einzelnen Herzschlag unter Einwirkung verschiedener Temperaturen ausgeworfenen Blutmenge zu, d. h. also der Volumsveränderung, welche das Herz beim Uebergange vom diastolischen zum systolischen Zustande aufweist. Es zeigt sich, dass die Verhältnisse hier nicht ganz so einfach und klar übersichtlich liegen, wie bei der Pulsfrequenz.

Zunächst ist die absolute Grösse des Pulsvolums nicht bei allen Herzen die gleiche, sondern unterliegt nach der Grösse und Individualität der Thiere viel grösseren Schwankangen, als die Pulsfrequenz. Diese zeigte bei den mittleren Temperaturen, die wir als Ausgangspunkt benutzten, selbst bei sehr verschieden grossen und kräftigen Thieren nur Differenzen von wenigen Schlägen, wäbrend die von den Herzen aufgenommenen and aufgeworfenen Blutquanta bedeutende Unterschiede aufwiesen. So betragen diese z. B. in einem Falle nur die Hälfte von den Mengen, die ein Herz in einem anderen Versuch förderte.

Ferner ist auf das Pulsvolum auch die Art der Herrichtung des Herzens fur den Versuch von Eunfluss; namentlich kommt es dabei auf die Wabl der Stellen an, an denen die Ligaturen angelegt werden. 
Auch die Ermüdung des Herzmuskels spielt beim Pulsvolum eine ganz andere Rolle wie bei der Frequenz. So gelang es in der Regel selbst bei langdauernden Versuchen, die Pulsfrequenz, nachdem sie sich mit den verschiedenen Temperaturen, denen das Herz ausgesetzt wurde, verändert hatte, wieder durch Herstellung der Ausgangstemperatur auf die ursprüngliche Zahl zurückzuführen. Dieses aber war. für die Richtigkeit der erhaltenen Resultate besonders beweisend. Dieselbe Bedingung bestrebten wir uns auch für das Pulsvolumen festzubalten. Hier aber gelang es nur bei einzelnen, sebr ausdauernden Herzen vollständig. Oefters mussten wir der Ermüdung Rechnung tragen und die sonst ja bei allen Versuchen gleichbleibende, als optimal erkannte Belastung etwas verringern, um, nach langer Dauer des Versuchs, bei der Ausgangstemperatur anch den ursprïnglichen Werth der ausgeworfenen Blutmenge wieder zu erhalten.

Folgende Beispiele mögen das Gesagte erläutern.

Das Herz einer Esculenta ergiebt bei optimaler Belastung:

6 h. $15^{0}, 30$ Pulse in der Minute, Vol. $=16$ Scalentheile. Das Herz wird bei gleichbleibender Belastung erwärmt (bei $24^{0} 52 \mathrm{Pulse}$ in der Minute, 11 Scalentheile n. s. w.) bis zur oberen Grenze, nach ganz kurzem Stillstand wieder abgekiihlt. Nun ergiebt sich:

$7 \mathrm{~h} .45 \mathrm{~m} .180,36$ Pulse in der Minute, Vol. $=8$ Scalentheile. Nach Senken des Flüssigkeitsspiegels des zufliessenden Blutes und der Ausflussöffnung um $8 \mathrm{~cm}$, also Verminderung der Belastung.

Volumen $=13$ Scalentheile.

Dies ist jetzt die optimale Belastung, weiteres Senken oder Wiedererheben vermindert das Volum des Pulsschlags. Während also nach $13 / 4$ stündl. Versuchsdauer bei einer der ursprünglichen Temperatur sebr naheliegenden die Pulsfrequenz sich wieder entsprechend verringert und der Ausgangszahl genähert hat, ist die frühere Grösse des Pulsvolums erst nach Verringerung der Belastung zu erzielen.

Die bei den verschiedenen Versuchen gewonnenen Zahlen lassen sich somit nicht unmittelbar vergleichen, sondern nur als proportionale Grössen verwerthen. Anfiihren wollen wir, dass wir als Maximalleistung einen Ausschlag am Volumeter von 29 Scalentheilen sahen, der einer in einer Herzcontraction geförderten Blutmenge von $0,145 \mathrm{~g}$ entsprach, gewiss eine grosse Leistung bei der geringen Masse des Organs.

Es lässt sich aber doch zeigen, dass auch die Grösse der Herzcontractionen sich mit der Temperatur nach einer gewissen Gesetzmässigkeit verändert, aber in einer gerade amgekehrten Weise wie bei der Frequenz. Denn das Minimum liegt hier bei den hohen Temperaturen, das Maximum dagegen bei dea 
niederen. Auch hier fehlen mir freilich die Bestimmungen für Temperaturen unter $3^{0}$; von diesem Punkte an aber nimmt die Grösse der Contractionen von Grad zu Grad allmählich ab. So regelmässig wie bei der Frequenz ist der Verlauf der Curve freilich nicht, wenigstens was die einzelnen Grade anbetrifft, wenn auch im Ganzen das gleiche Verbältniss bei allen Versuchen mehr oder weniger dentlich hervortritt. Nur ungefähr lässt sich so angeben, dass im Durchschnitt die Grösse der Contraction sich von einem Grad zum anderen etwa um 1/10 der Grösse verändert, die das Herz bei circa 21 ${ }^{\theta}$, der gewöhnlichen Temperatur, aufweist: die Abnahme nach den höheren Graden zu ist meist wohl etwas bedeutender als die Zunahme nach den kälteren. Die gestrichelte Linie der Figur 2, bei der die Grössen des Ausschlags im Volumeterrohr oder die von einer Contraction geförderte und gewogene Blutmenge als Ordinaten aufgetragen sind, zeigt, dass auch diese Curven im Grossen und Ganzen einen geradlinigen Verlauf haben. Die obere Grenze fällt natïrlich mit der Grenze der Herzthätigkeit überhaupt zusammen und liegt also etwa bei $32^{\circ}$ mit oft schon früher beginnenden Unregelmässigkeiten. Auch den von $\mathrm{Cy}_{y}$ on beschriebenen Zustand kurz vor dem völligen Stillstand des Herzens, das Delirium cordis, Herzperistaltik, sahen wir mehrmals. Von vielen Stellen gehen nämlich fortwährend Contractionsimpulse aus, die wellenförmig über das ganze Herz verlaufen, während kein Tropfen Blut gefördert wird. Man möchte sagen, hier sei die Frequenz der Contractionsimpulse $=\infty$, die Grösse der hervorgerufenen Contraction aber $=0$ (und folglich auch die Arbeitsleistung $=0 . \infty=0$ ).

In den oberen Grenztemperaturen sind die Volumina, die an der Scala abgelesen werden, meist sehr klein (3-5 Theilstriche). Hier finden sich auch, entsprechend den Unregelmässigkeiten in der Frequenz, die oben erwähnt sind, Ungleichmässigkeiten in dem Umfang der einzelnen Contractionen, nämlich ab und zu Palsvolumina von uberraschender Grösse. Und zwar stehen diese beiden Vorkommnisse im Zusammenhang: der Schlag, der nach längerer Pause auftritt (vor dem also, nach unserer Anschauung, eine Contraction aus. gefallen ist), übertrifft stets an Grösse bei Weitem die übrigen. Es scheint sich demnach zu bewahrheiten, dass die Grösse der Pausen bestimmend ist fiur den Umfang der Contractionen. Doch ist es zweifelhaft, ob dies Verhältniss sich so auffassen lässt, dass die Contractionen weniger umfangreich ausfallen, wenn dem Herzen zwischen den einzelnen Schlägen die Zeit fehlt, sich wieder auszudehnen, ein Satz, den auch Cy on nach den Ergebnissen seiner Versuche bestreitet. 
Dass tiberhaupt der Umfang der Contractionen, also der Unterschied zwischen der Länge der Muskelfasern im diastolischen und systolischen Zustande, bei höheren Temperaturen geringer ist, dürfte jedenfalls, wenigstens am isolirten Herzen, nicht in nervösen Einfliissen, sondern ,in den veränderlichen Eigenschaften der Muskelfasern selbst liegen", - wie dies Bowditsch 1) ausführlich erwiesen hat, auf Grund des Gesetzes, für das auch Kronecker eintritt, dass der minimale Reiz zugleich der maximale für die Contraction des Herzens ist.

Die Grösse der Contraction der Fasern könnte nun zunächst dadurch eine geringere werden, dass das erwärmte Herz in der Diastole zwar gleich gross oder wohl gar grösser ist, als in derselben Phase seiner Thätigkeit bei niederer Temperatar, dass es aber bei der Systole nur in einen schwächeren Contractionszustand geräth, also ausgedehnter bleibt, als das systolisch contrahirte kalte Herz: so wäre die Differenz zwischen dem Herzvolumen in Systole und Diastole geringer beim kalten, als beim warmen Herzen, demnach das Pulsvolumen kleiner. Dafür scheint die Angabe Cyon's zu sprechen, dass bei höheren Temperaturen eine grössere Ausdebnbarkeit der Herzwandungen vorhanden ist, wofür er als Beweis anführt, dass das Quecksilber eines mit dem Herzen verbundenen Manometers auch in der Diastole bei höheren Temperaturen tiefer steht, als bei niederen. Unsere Versuchsanordnung erlaubte leider eine Nachuntersuchung für diese Verhältnisse nicht.

Weit einfacher und natürlicher lässt sich aber die Erscheinung: durch die Annahme erklären, dass das Volum des ausgedehnten Herzens in der Diastole bei höheren Temperaturgraden kleiner ist, als bei niederen; so kann dann auch der Effect der systolischen Contractionen nur ein geringerer werden. Für diese Deutung spricht eine Beobachtung von Schmulewitsch 2). Derselbe fand, dass die Wärme eine rein physikalische Wirkung auf den lebenden Froschmuskel austibt, und zwar gerade in den uns interessirenden Temperaturen von $2-28^{\circ}$; Die Muskelsubstanz des völlig lebensfrischen Muskels würde nämlich beim Erwärmen kürzer und beim Abkühlen länger.

Die Frage, zu deren Besprechung wir jetzt übergehen, bietet,

1) Ueber die Eigenthümlichkeiten der Reizbarkeit, welche die Muskelfasern des Herzens zeigen. Arbeiten aus der physiologischen Anstalt zu Leipzig. 1872.

2) Zur Muskelphysik und Physiologie 1867. Centralblatt f. die modicinische Wissensch. 1867. S. 81 . 
wenigstens wenn wir die Rückwirkung anf den ganzen Organismus des Thieres berïcksichtigen, das meiste Interesse: inwiefern nämlich die eigentliche Arbeitsleistung des Herzens in der Zeiteinheit von Aenderungen der Temperatur beeinflusst wird, d. b. wieviel Blut das Organ während gleicher Zeiten bei verschiedenen Wärmegraden in die arteriellen Bahnen zu werfen vermag. Es handelt sich im Folgenden natürlich nur um den optimalen Werth der Arbeit, die das isolirte Herz unter günstigen Bedingungen leisten kann. Dazu gehören besonders genügende Ernährung, optimale Belastung; gut schliessende Klappen, resp. bei unserer Versuchsanordnung richtig functionirende Ventile, endlich eine gentigende Blutzufuhr in das Herz. Besonders leicht ergeben sich bei dem William'schen Apparate fehlerbafte Resultate, wenn die Ventile entweder nicht genügend schliessen, so dass Blut bei der Systole in die venöse Seite des Kreislaufs regurgitiren kann, oder zu fest angezogen sind, so dass dann das Herz ungenügend gefüllt sich contrahirt.

Bei den Versuchen wurde nun bald die in der Minute ausgeworfene Blutmenge direct gewogen, bald wurde sie aus der Zahl und dem Volumen der Herzcontractionen berechnet (p. t. ) - Beides ergab übereinstimmende Resultate. Die eigentliche Arbeitsleistung ist dann durch das Product dieser Grösse mit der Hubhöhe (p. t. h.) dargestellt. Diese ist aber (vgl. die citirte Arbeit von Dreeser) bei der üblichen Anordnung des William'schen Apparates nur der Höhenunterschied zwischen dem Niveau der Flüssigkeit im Zuflussgefäss und der Ausflussöffnung, d. h, wenige Centimeter und stets die gleiche. So konnten wir diesen Factor ganz eliminiren, da es uns nicht auf Feststellung der absoluten Grösse der Arbeitsleistung ankam, sondern unr auf das Erkennen der Veränderungen, die diese bei versehiedenen Temperaturen erleidet.

Schon wenn man die Curven des Volums der Hercontractionen und der Zahl combinirt, ergiebt sich, dass bei der den Temperaturgraden nach entgegengesetzten Lage der Maxima und Minima und dem fast geradlinigen Verlauf der Curven die Arbeitsleistung ziemlich parallel der Abscissenaxe verlaufen muss. Und dieses Resultat bestätigen alle gelungenen Versuche mit gut arbeitenden Herzen.

Wir verweisen auf die ausgezogene Linie der Fig. 2, welche Curve den Einfluss der Temperatur auf den Natzeffect der Herzthätigkeit erkennen lässt, - die Arbeitsleistungen in der Zeiteinheit sind hier als Ordinaten aufgetragen. Natürlich konnten wir die Werthe aus den verschiedenen Versuchen nur in ibrer Relation zu einander berïcksichtigen. 
Die Arbeitsleistung des Froschherzens ist also von $3^{0}$ an - und nichts lässt annehmen, dass es bis zu der wenige Grade niedriger liegenden Grenze des Herzschlags überhaupt anders sei - b is zuder höchsten Temperatur, in der das Herz sich zu contrahiren vermag, fast die gleiche. In den der oberen Grenze am nächsten liegenden, letzten Graden können freilich die Unregelmässigkeit der Frequenz und des Pulsvolumens auch die Arbeitsleistung beeinflussen; aber, wenn die Unregelmässigkeiten nicht sehr hochgradige sind, so bleibt das Ergebniss für die geleistete Arbeit doch das gleiche, da, wie erwähnt, die auf die ausgefallenen folgenden einzelnen Contractionen um so ausgiebiger sind, und so eine Compensation eintritt.

Immerbin liess sich ein, wenn auch unerhebliches, Ansteigen und Abfallen der Arbeitsleistung nachweisen, und zwar lag das Optimum bei unseren Versuchen etwa bei 20-22. Von da aus sank die Leistung ganz allmählich nach beiden Seiten ab. Betrug dieselbe, um eine rohe Durchschnittszahl anzugeben, im Optimum bei einer Hubhöhe von $1-2 \mathrm{~cm} 4 \mathrm{~g}$ in der Minute gefördertes Blutquantum, so sank sie an der oberen und unteren Grenze etwa auf $3 \mathrm{~g}$, wenigstens bei den bestgelingenden Versuchen. Diese fur die Arbeitsleistung optimale Temperatur $\left(20-22^{0}\right)$ war aber die Temperatur des Zimmers, in dem der Froschbehälter stand, während der Jahreszeit, in der diese Versuche angestellt wurden. Wurden die Frösche, wie dies gelegentlich geschehen ist, 24 Stunden lang in einer Temperatur von $30^{\circ}$ gehalten, und ihre Herzen, ohne sie abzukühlen, in den Apparat gebracht, so zeigte sich, dass dieselben entschieden mit für diese hohen Temperaturen auffallend grossem Nutzeffect arbeiteten.

Wir sehen also, wie sich der Frosch im Allgemeinen als Kaltblüter der Temperatur der Umgebung anzupassen, so auch sein Herz, dies für das Leben so wichtige Organ sich in seiner Function auf die verschiedenen Wärmegrade einzurichten vermag. Jede Verände. rung in der Temperatur wirkt wohl etwas schädigend auf die Arbeitsleistung seines Herzens, aber doch in unwesentlicher Weise: denn was der Herzschlag an Frequenz verliert, gewinnt er an Ausgiebigkeit der Contraction, und umgekehrt. Also, um ganz kurz zusammenzufassen :

Von der unteren (etwa $0^{\circ}$ ) zur oberen Grenze (ungefähr 320 der Herzthätigkeit steigt gleichmässig die Frequenz des Herzschlages, sinkt ebenso ihr Volumen, und bleibt die in derZeiteinheit geleistete Arbeit ziemlich die gleiche und wird nur etwas optimal, d. h. grösser 
bei der Temperatur, bei der der Frosch gerade zu leben gezwungen ist (hier 20-220).

Was endlich das Verhalten der absoluten Kraft des Herzmuskels bei verschiedenen Temperaturen anbelangt, so ist dasselbe einfach darzustellen. In den Grenzen, in denen überhaupt das Herz thätig ist, bleibt die absolute Kraft desselben fast unverändert. Ein geringes Optimum etwa in denselben Temperaturgraden, wo das der Arbeitsleistung liegt, und ein unbedeutendes Absinken gegen die obere und untere Grenze scheint constant zu sein. Dies zeigt die unterste Curve der Fig. 2, bei der die Höhen, bis zu der das Herz die Blutsäule zu heben, resp. ihren Druck zu überwinden vermag, in Centimetern als Ordinaten aufgetragen sind.

Noch ein Verbältniss möchte ich kurz erwähnen. Dreeser hat gefunden, dass die von dem einzelnen Pulse ausgeworfene Blutmenge bei einer bestimmten Belastung (optimale Belastung) am grössten ist. Die Grösse der optimalen Belastung ist je nach dem Herzen etwas verschieden, ohne sehr beträchtlich zu variiren (zwischen 20 u. $30 \mathrm{~cm}$ ). Diese Grösse schwankt aber auch für das einzelne Herz mit den verschiedenen Temperaturen. Und zwar gevügt bei niederen Temperaturen eine geringere Belastung, um das höchste Pulsvolum herzustellen, als bei böheren. Ist ja hier die Contraction schon ohnehin dafür geeignet, ein grosses Volumen zu ergeben. Ueber etwa $20^{\circ}$ hinaus die kleinen Pulsvolumina durch Vermebrung der Belastung zu vergrössern, gelingt aber nicht, hier endet eben die Kraft des Herzens. Berticksichtigen muss man auch, wie sehr hier die Ermüdung täuschen kann: das ermüdete Herz erheischt selbstverständlich eine geringere Belastung, als optimale.

Nach den Ergebnissen der angeführten Untersuchungen musste man die Frage aufwerfen, wie es denn möglich sei, dass die Frösche jene höheren, an sich den Herzschlag vernichtenden Temperaturen, denen sie ja in der Natur nicht selten ausgesetzt sind, ertragen. Da das isolirte Herz schon um $30^{\circ}$ herum unregelmässig zu schlagen anfing und zwischen 32 und $35^{\circ}$ stets zum Stillstand kam, so hätte doch auch der Frosch, an dem man vorauszusetzen gewohnt ist, dass er als Kaltblüter stets die Temperatur der Umgebung annimmt, bei höheren Wärmegraden nicht existiren können. Dennoch lebt im heissen Sommer an der Luft auch eine R. esculenta ganz gut bei Temperaturen, die $32^{\circ}$ übersteigen.

Noch erwähnen wollen wir hier, dass auch andere Organe des Frosches bei dieser Temperatur ihre Leistungsfähigkeit verlieren: so 
verändern sich nach Harless ${ }^{1}$ ) zwischen 34 u. $37^{\circ} \mathrm{C}$. die Nerven sehr wesentlich, werden wenig leistungsfähig.

Dass das Herz in situ nicht etwa höhere Temperaturen vertragen könne, als das isolirte Organ, war von vornherein sehr wahrscheinlich, und es liess sich auch zeigen, dass unter bestimmten Bedingungen, auf die wir später noch zurückkommen, die Frösche bei Temperaturen, die jedenfalls nur ganz wenig höher lagen, als $32^{\circ}$ (höchstens $35^{\circ}$ ), ausnahmslos bald zu Grunde gingen.

So blieb nur übrig, daran zu denken, dass der Frosch, wenn er thatsächlich höhere Lufttemperaturen zu uiberdauern vermag, in gewissen Grenzen seine Temperatur niedriger als die Aussentemperatur zu erhalten im Stande ist. Wenn wir uns hier an den analogen Vorgang bei homoiothermen Thieren erimnern, so ist dies jedenfalls nicht ganz unwahrseheinlich. Nor fragt es sich dann, in welcher Weise es bei dem Frosch möglich sei, dass seine Körpertemperatur gegenüber der Wärme der Umgebung geringer bleibe.

Der eine Factor, der den Warmblitern in hohem Grade hilft, sich kühler zu erhalten, die Verminderung des Stoffwechsels, kann hier nicht in Betracht kommen. Der Stoffwechsel ist ja beim Frosch so gering, dass seine etwaige Verminderung eine merkbare Einschränkung der Wärmeproduction jedenfalls nicht geben kann. Auch eine vermehrte Wärmeabgabe, indem also etwa das Blut in erweiterten Gefässen und in grösserer Menge an die Haut gelangt, ist ausgeschlossen, da wir ja als Bedingung haben, dass die Temperatur der Umgebung, höher ist, als die des Thieres.

So mïsste denn also der Frosch sich selbst Kälte erzeugen, und dies ist natiurlich einzig und allein dadurch möglich, dass eine starke Verdunstung von seiner Oberfläche, von der Haut and auch von den Lungen sich einstellt. Ist dies aber in der That der Fall, regulirt der Frosch auf diese Weise seine Körpertemperatur, so muss es gelingen, wenn wir das Thier unter Bedingungen setzen, die die Verdunstung begünstigen, auch bei hohen Temperaturen das Herz des Frosches functionsfähig zu erhalten, das Leben desselben zu bewahren. In dieser Richtung wurden deshalb noch einige Versuche angestellt.

Im Allgemeinen nimmt der Froseb bei seiner geringen Masse rasch die Aussentemperatur an. Steckten wir einem Frosch ein kleines Thermometer in den Magen und hielten das Thier während der

1) Ueber den Einfluss der Temperaturen und ihrer Schwankungen auf die motorischen Nerven. 1860. Zeitschr. f. rationelle Medicin. 3. Reihe. Bd. VIII. 
Manipulation unter dem Wasser des Froschkastens, so zeigte dieses 20. Nahmen wir aber den Frosch heraus und hielten ihn in der Hand, so stieg das Thermometer in der allerkürzesten Zeit um 6-70, brachten wir ihn dann unter die Wasserleitung, sank die Temperatur desselben sofort wieder.

Für die weiteren Versuche, bei denen die Thiere längere Zeit das Thermometer in ihrem Körper bebalten sollten, war das Einführen in den Magen nicht zweckentsprechend, da es die Frösche sehr störte und in der Athmung behinderte. Es wurde deshalb ein kleines Thermometer in den Anus eingeführt, bis in die Nähe des Herzens vorgeschoben und mit einem durch die Haut gezogenen Faden befestigt. So konnten die Frösche sich ganz unbehindert bewegen und bequem athmen.

Um nun die Thiere in einer stets trockenen Luft zu haben und diese längere Zeit auf einem höheren Wärmegrad constant erhalten zu können, trafen wir folgende Einrichtung. Eine geräumige Blechwanne, in der sich durch das zwischen ihren doppelten Wänden befindliche warme Wasser bequem jede Temperatur berstellen liess, wurde mit einer Glasscheibe, aber nicht luftdicht bedeckt. In die Wanne wurde eine Glasglocke mit Tubus und einer durchlässigen Bodenplatte eingesetzt und durch Holzbänkchen mit Filz gegen jede directe Wärmeleitung vom Boden der Wanne aus geschützt. Durch den Tubus führte eine Glasröhre, die weiter durch die Wand der Wanne ging und durch einen starken Gummischlauch mit einem Aspirator in Verbindung stand.

Durch diese Glasglocke wurde dann während des Versuches mittelst des Aspirators ein kräftiger, trockener Luftstrom mit ziemlicher Geschwindigkeit hindurchgesaugt. So gelangte also nur die vorher innerbalb der Wärmwanne befindliche und dort erwärmte Laft in die Glasglocke. Dicht vor dem Eintritt in die Glasglocke und kurz nach ibrem Austritte wurde die Temperatnr der Luft gemessen, und Alles so regulirt, dass beide Thermometer möglichst genau denselben Grad zeigten. Der Frosch, dem wiederum ein kleines Thermometer in den Anus gesteckt war, erhielt $2 \mathrm{ccm}$ physiologischer Kochsalzlösung in den Lymphsack, um ibn vor Austrocknung zu schïtzen, und wurde in die Glasglocke gesetzt. Er befand sich so in einer Luft, die auf der für jeden Versuch gewünschten Temperatur constant gehalten wurde, trocken war und stets ernenert wurde, so dass sie das Versuchsthier selbst durch seine Absonderung nicht mit Wasserdampf zu schwängern vermochte: die geforderten günstigen Bedingungen für die etwaige Temperaturregulirung waren hergestellt.

Wir geben hier einige Versuchsprotokolle: 
Ueber den Einfluss d. Temperatur auf die Thătigkeit des Froschberzens. 381

I. Esculenta. Die Temperatur der Luft beträgt $42^{\circ}$.

Um 5 h. $15 \mathrm{~m}$. wird der Frosch, dessen Körpertemperatur (entsprechend der im Froschbehälter herrschenden) $20^{\circ}$ ist, in die Glasglocke eingesetzt.

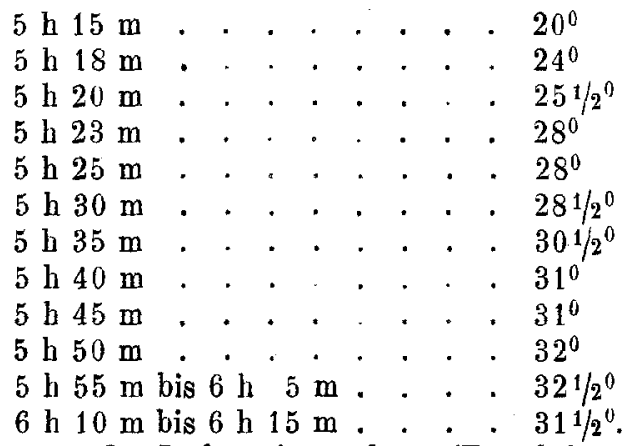

Die Temperatur der Luft steigt auf $44^{\triangleleft}$ (Frosch bewegt sich stark)

$6 \mathrm{~h} 20 \mathrm{~m}$ bis $6 \mathrm{~h} 30 \mathrm{~m}$. . . . $33^{0}$

$6 \mathrm{~h} 35 \mathrm{~m}$ bis $7 \mathrm{~h}-\mathrm{m}$. . . $33 \frac{1}{2}$.

Die Temperatur der Luft steigt auf $45^{\circ}$ (Frosch munter, springt)

$7 \mathrm{~b} 5 \mathrm{~m}$. . . . . . . $34^{0}$

$7 \mathrm{~h} 10 \mathrm{~m}$ bis $7 \mathrm{~h} .30 \mathrm{~m}$. . . $34 \frac{1}{10}$.

Der Versuch wird abgebrochen. Der Frosch ist völlig normal, da die wohl nicht bedeutende Schädigung, die sein Herz erlitten, das Thier noch nicht alterirt.

II. Esculenta mit einer Körpertemperatur von $21^{\circ}$, die Temperatur der Luft wird constant auf $50^{\circ}$ gehalten.

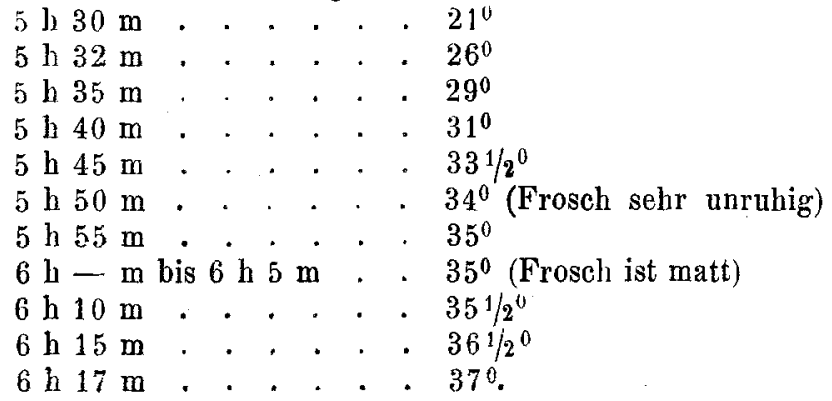

Der Frosch ist sehr matt. Die Temperatur hält sich noch längere Zeit anf dieser Höhe, dann Tod des Thieres.

Beide Beispiele zeigen, wie sehr rasch die Körpertemperatur in einer wärmeren Umgebung zunächst ansteigt, in 10 Minuten im ersten Fall um $8^{\circ}$, im zweiten um $10^{\circ}$, and wie dann eine bedeutende Verlangsamung dieser Steigerung eintritt. Im ersten Falle (bei $42^{\circ}$ Lufttemperatur) steigt die Temperatur des Frosches von 28 auf $31^{0}$ noch 
in 15 Minuten, zwischen $31^{\circ}$ und $32^{1 / 2^{0}}$ bewegt sie sich dagegen 35 Minuten lang, nach einer kleinen Erhöhung der Aussentemperatur verharrt sie zwischen 33 and $33^{1 / 2}{ }^{0} 40$ Minuten und zwischen 34 und $34 \frac{1 / 20}{20}$ Minuten und jedenfalls noch länger, wäre der Versuch nicht abgebrochen worden.

Und im zweiten Falle, wo die höhere Aussentemperatur von $50^{\circ}$ der Regulation der Körperwärme des Thieres jedenfalls bedeutende Schwierigkeiten entgegenstellt, dauert immerbin, während das Ansteigen der Temperatur von $21-31^{\circ}$ in 10 Minuten vor sich geht, der Uebergang von $31-331 / 2^{0} 5$ Minuten, von $331 / 2-35^{0} 10$ Minuten, von 35-35 $\frac{1 / 2}{2} 15$ Minuten. Dann freilich geht - das Thier ist matt - die Temperatur auf $361 / 2^{0}$ und $37^{\circ}$, und der Frosch stirbt. Ganz ähnliche Resultate erhielten wir bei anderen Versuchen, sobald wir für genügende Zuführung trockener Luft sorgten. Wurden die Temperaturen niedriger genommen, als bei den angeführten Versuchen, etwa $40^{\circ}$ oder darunter, so vermochten die Frösche und ihre Herzen ohne jede Schädigung zu bleiben; sie verharrten auf ungefähr $32-33^{0}$.

Diese Thatsachen sagen also zunächst nur, dass, wie jeder todte oder lebende Körper, der Frosch in wärmerer Umgebung zunächst rasch erwärmt wird; dass aber, je geringer die Temperaturdifferenz wird, je mehr sich die Temperatur des Thieres der der Umgebung nähert, um so langsamer die weitere Erwärmung vor sich geht. Ebenso selbstrerständlich ist es, dass hier, bei der stets feuchten Haut des Thieres, die Verdunstung dafür mit in Betracht kommt, welche Temperatur der Frosch zeigt.

Es kam uns aber gerade darauf an zu sehen, in welchem Grade diese Verdunstung einwirke, und ob sie ausreiche, den Frosch in hober Temperatur am Leben zu erhalten. Und so ergab sich ein wohl bemerkenswerthes Resultat. Selbst bei Aussentemperaturen von $45^{0}$ bleibt die Temperatur des Frosches Stunden lang um mehr als $10^{\circ}$ tiefer als die der Luft, - so bedeutend ist also bei dem kleinen Thiere der Einfluss der durch die Verdunstang der Hautflüssigkeit erzeugten Kälte. Bei Temperaturen, die tiefer liegen (etwa $40^{\circ}$ und darunter) - die also factisch überhaupt nur in Frage kommen -, ist dieser Factor also gewiss genügend, um die Temperatur des Frosches gegenüber der höheren Aussentemperatur ausreichend niedrig (etwa $32^{\circ}$ ), also in den Grenzen, in denen das Thier zu existiren vermag, za halten.

Noch anführen wollen wir, dass bei Fröschen, denen das Thermometer durch den Mund in den Magen eingeführt war, die also in 
Ueber den Einfluss d. Temperatur auf die Thätigkeit des Froschherzens. 383

ihrer Athmung behindert waren, die Temperatur des Körpers in den kritischen Graden rascher der der Umgebung gleich zu kommen schien. So scheint also die Ausdunstung durch die Lungen mit einzuwirken, und glaubten wir auch öfters eine grössere Frequenz der Athmung wahrzunehmen.

Nachdem diese Versuche zu dem erwähnten Ergebniss geführt hatten, musste man, um sicher zu gehen, noch prïfen, ob denn unter anderen Umständen, wenn die Bedingungen ungünstige waren, die Thiere wirklich zu Grunde gingen. Die Frösche mussten also in eine recht durchfeuchtete, erwärmte Luft gebracht werden. Dies erlaubte eine andere Versuchsanordnung. In ein Glasgefäss, das zum Theil mit Wasser gefiullt war, wurde der Frosch (mit dem Thermometer im Darm) auf einen Gazeboden gesetzt, so dass er nicht mit dem erwärmten Wasser in Berührung kam. Vorher hatten wir die Luft möglichst mit Wasserdampf geschwängert, indem wir einen Luftstrom durch das Wasser und das Gefäss langsam streichen liessen.

$Z$ u einem Versuch wurde z. B. die Luft auf $36-38^{\circ}$ erwärmt erhalten, und der Frosch, der eine Temperatur von $20^{\circ}$ zeigte, hineingebracht. Schon nach $3 / 4$ Stunden war das Thier ganz matt, nach $11 / 4$ Stunde todt, das Herz schlug zwar noch bei Eröffnung des Brustkorbs, aber ganz unregelmässig, peristaltisch und stand bald völlig still. In dieser mit Feuchtigkeit gesättigten Luft, in der eine Verdunstung jedenfalls nur in sehr geringem Umfange möglich war, tritt also bei einer (gegenüber den oben erwähnten Versuchen nicht hohen) Lufttemperatur von $38^{\circ}$ der Tod schon nach 11/4 Stunde ein, - während in dem oben erwähnten Versuche ein mehrständiges Verweilen bei $42-45^{\circ}$ das Thier ganz unberïhrt lässt.

So glauben wir, ist es erwiesen, dass der Frosch einzig und allein durch eine vermehrte Wasserverdunstung und die so erzeugte Kälte seine Temperatur gegenüber der umgebenden Luft herabzusetzen, zu reguliren vermag, sobald sich dieselbe den Wärmegraden nähert, die sein Herz und damit sein Leben gefährden.

Der Frosch, obwohl er ein Kaltblüter, ist also - dies ergiebt sich aus Allem angeführten - bei sehr weit auseinanderliegenden Temperaturen der Umgebung lebensfähig. Von etwa $0^{0}$ bis ungefähr $32-35^{\circ}$ vermag sein Herz die nothwendige Arbeit zu leisten. Steigt aber die Aussentemperatur höher, so wird der Frosch gewissermaassen poikilotherm; sein Körper, sein Herz bleibt innerhalb der ertragbaren Temperatur, also arbeitsfähig. Nur dürfen die sonstigen Bedingungen nicht ungünstige sein: die Luft nicht feucht, oder die hohe Temperatur zu lange einwirken oder excessiv hoch werden. 
384 XVIII. Fuatow, Ueber den Einfluss der Temperatur u. s. w.

Zum Schluss ist es dem Verfasser eine angenehme Pflicht, Herrn Prof. Dr. Schmiedeberg, Director des pharmakologischen Institats zu Strassburg, für die Anregung zu dieser Arbeit und gütige Unterstützung bei der Ausführung derselben, sowie Herrn Privatdocenten Dr. Jakoby, I. Assistenten des Instituts, für seine bereitwillig gewährte Beihtilfe bei der Anstellung der Versuche seinen ergebenen Dank auszusprechen. 